\title{
A novel handover scheme using torch nodes and adaptive measurement aggregation mechanism to improve QoS in high-speed railway communication
}

\author{
P. J. Pramod ${ }^{1}$ • B. C. Jinaga ${ }^{2}$ (D)
}

Received: 24 April 2017/Revised: 9 November 2017/Accepted: 15 November 2017/Published online: 1 December 2017

(C) The Author(s) 2017. This article is an open access publication

\begin{abstract}
Technological advancement in the field of transportation and communication is happening at a faster pace in the past few decades. As the demand for high-speed transportation increases, the need for an improved seamless communication system to handle higher data traffic in a highly mobile environment becomes imperative. This paper proposes a novel scheme to enhance the quality of service in high-speed railway (HSR) communication environment using the concept of torch nodes (TNs) and adaptive measurement aggregation (AMA). The system was modeled using an object-oriented discrete event simulator, and the performance was analyzed against the existing single-antenna scheme. The simulation results show that the proposed scheme with its minimal implementation overhead can efficiently perform seamless handover with reduced handover failure and communication interruption probability.
\end{abstract}

Keywords High-speed railway · Cellular wireless technology · Long-term evolution · Mobile relays · Handover scheme $\cdot$ Quality of services

P. J. Pramod

pramodpj@cdac.in

B. C. Jinaga

jinagabc@gmail.com

1 Electronic System Design and Manufacturing (ESDM) Group, Centre for Development of Advanced Computing (CDAC), Plot No. 6 and 7, Hardware Park, Hyderabad, Telangana 500005, India

2 Electronics and Communication Engineering Department, Jawaharlal Nehru Technological University Hyderabad, Kukatpally, Hyderabad, Telangana 500085, India

\section{Introduction}

The twenty-first century is experiencing tremendous growth of data and Internet services [1] to meet the significant rise in socioeconomic developmental needs [2] and user demand. Smart devices demanding cellular wireless connectivity are increasing exponentially and have crossed the current world population [3]. Wireless networks are also enhancing its capability to handle more devices, users and larger data traffic.

Advancement in high-speed railway (HSR) transportation has resulted in more passengers transiting through railway networks [4]. HSR is currently becoming more and more popular, and this mode of public transportation is preferred in several countries due to its speed, safety, energy efficiency and larger passenger handling capacity [5-9]. The demand for high-quality video, voice and data over broadband wireless communication in an HSR environment has thrown open various limitations of cellular wireless technology.

The LTE-Advanced standardization was through 3GPP release 10 version of specification has introduced longterm evolution-advanced (LTE-Advanced) $[10,11]$ which provides better bandwidth, higher efficiency and enhanced intercell interference coordination (eICIC). The data rates were enhanced to $1 \mathrm{Gbps}$ (downlink, DL)/500 Mbps (uplink, UL) for low mobility application and $100 \mathrm{Mbps}$ (DL)/50 Mbps (UL) for high mobility application [12] along with an increase in requirements of peak spectral efficiency to $30 \mathrm{bps} / \mathrm{Hz} \quad$ (DL)/15 bps/Hz(UL). This enhancement has put forward significant implementation challenges to HSR system designers [13].

Figure 1 shows the architecture of LTE-Advanced with two distinct components: (1) evolved universal terrestrial radio access network (E-UTRAN) and (2) evolved packet 


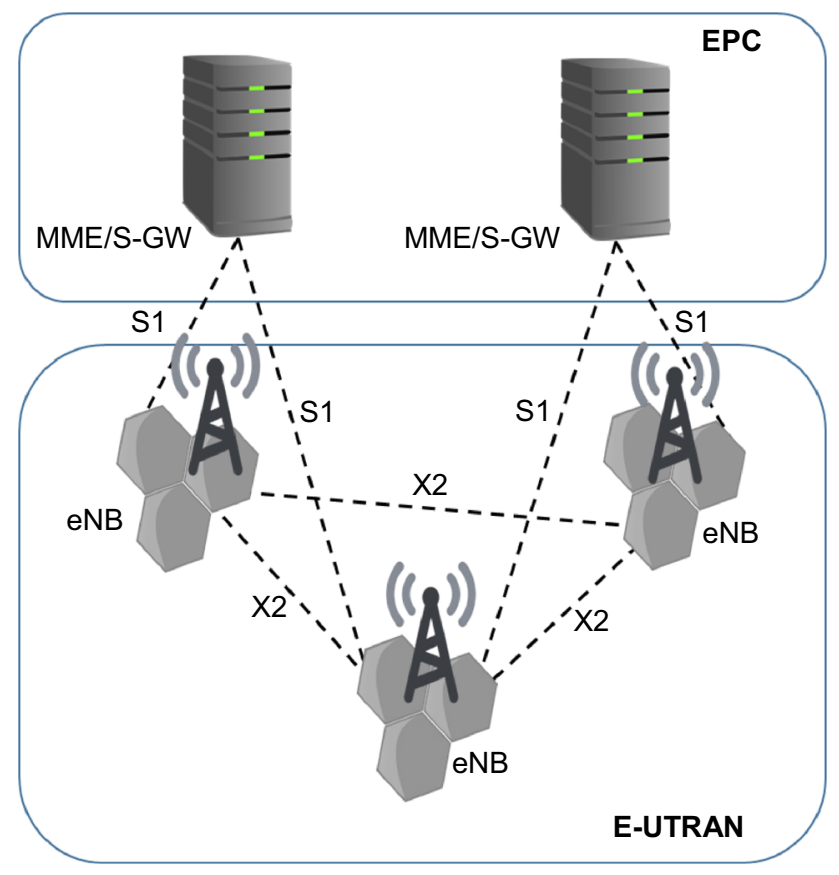

Fig. 1 LTE-Advanced network architecture

core (EPC) [14]. The EPC consists of mobility management entity (MME), serving gateways (S-GW) and packet data network gateway (P-GW) which provide services like signaling, handover, security, whereas the E-UTRAN incorporates multiple interconnected evolved NodeBs (eNBs) [15]. These eNBs are interconnected using X2 interface, whereas they are connected to EPC through S1 interface.

A highly mobile HSR scenario involves long narrow strips, bridges, viaducts and directional antennas with reduced cell coverage. The deployment of wireless networks for such a scenario is challenging due to increased handover (HO) frequency resulting in significant performance degradation [16]. To make the condition worse, the implementation of HO in LTE-Advanced is a hard HO which requires breaking the radio link with source before connecting with target eNB. Frequent HOs in such traditional networks would increase the network load and power consumption of user equipment (UE), thereby affecting the overall quality of services (QoS) [17]. Group HO, frequent $\mathrm{HO}$ at short time intervals, Doppler frequency shift, penetration path loss and fading in propagation characteristics due to shielded carriage of trains, viaduct, hilly terrain and tunnel $[18,19]$ are major concern for system designers in a high-speed scenario.

In order to improve communication in a long-distance HSR scenario, leaky coaxial cable (LCX)-based radio communication system [20] was introduced to provide better bandwidth and efficient frequency reuse. The need for repeaters to compensate for transmission losses, high maintenance cost and large deployment cost limits the acceptance of LCX system for high-speed train-ground information transmission. Radio-over-fiber (RoF)-based system [21, 22] with remote antennal units (RAU) deployed along railway lines to transmit data wirelessly to the onboard unit is another promising technology for HSR communication. Support for higher bandwidth, cost-effectiveness, reduced power consumption due to linear radiated antenna, and reduced $\mathrm{HO}$ are the major features of RoF technology [23, 24].

Introduction of wireless local area networks (WLAN) to complement satellite communication in HSR scenario by M. Han et al. [25] was another significant work to overcome the limitation of $\mathrm{HO}$ and coverage. However, the bandwidth limitation, inherent delay in satellite-based system, blind spots, initial investment cost and requirement of line of sight have confined the scalability of this model in HSR.

A seamless HO scheme based on dual-link architecture was proposed by Lin Tian et al. [26]. The system with two antennas in the front and rear takes advantage of bi-casting and dual antennas to implement soft $\mathrm{HO}$ with reduced overhead and latency.

3GPP TR-36.836 has introduced mobile relays as base station [27] to circumvent the connectivity issues in HSR. These multi-RAT relays would provide wireless connectivity and services to onboard UE. Such a shift in connectivity maintenance from portable mobile devices to relays has significantly reduced the UE power consumption [28], signal storming or group HO [29], Doppler frequency shift and penetration path loss [30]. Though mobile relays have improved the performance between UE and eNB, the $\mathrm{HO}$ issues limiting the performance and reliability persist. Introducing relays as an intermediate node has only shifted the $\mathrm{HO}$ issues from UE to relay nodes. Increased load on these nodes has further deteriorated the packet latency, QoS, frequent $\mathrm{HO}$ and resilience capability of the overall system.

An effective two-link architecture based on distributed antenna system (DAS) and mobile relay was introduced by Liu et al. [31]. Onboard devices in this system are connected to train relay nodes which are further interfaced to remote central station using multiple RAUs based on RoF technology. However, the proposed system has neglected the period of $\mathrm{HO}$, which significantly affects the performance in real networks [32]. An enhanced HO scheme [33] was proposed using special control mobile relays (cMRs) equipped in front of a train and several mobile relays were distributed across the train. This scheme also proposed an enhanced measurement procedure to reduce the duration of cMR signal measurement based on the direction of movement. The cMR-based scheme is effective in reducing the signaling overhead and HO interruption time, whereas 

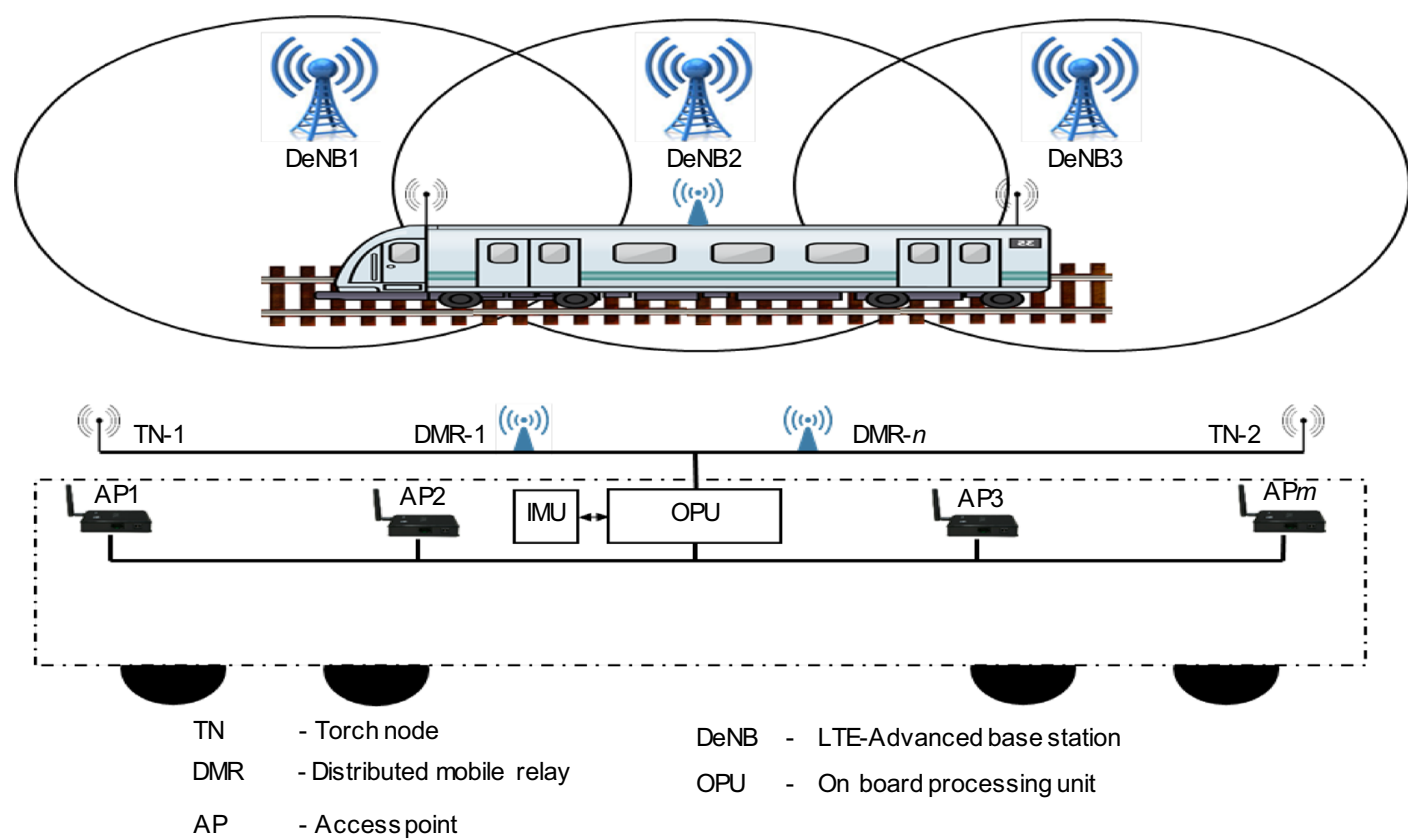

Fig. 2 System architecture

failure of $\mathrm{HO}$ at cMR would inherently affect the overall connectivity of the system.

In this paper, in order to overcome the limitations of existing systems, we introduce a novel $\mathrm{HO}$ scheme to enhance the QoS in an HSR communication environment by proposing the concept of torch nodes (TNs) and mobility-based adaptive measurement aggregation (AMA) to improve the existing $\mathrm{HO}$ mechanism. By introducing this approach through a distributed mobile relay (DMR)based architecture, we also address the resilience capability in HSR systems.

The rest of the paper is organized as follows. In the next section, we present our proposed solution, describing the system architecture and operational procedures. Various stages of the proposed $\mathrm{HO}$ scheme are elaborated in Sect. 3. Section 4 details the AMA mechanism. Implementation of simulation model and performance analysis will be carried out in Sect. 5. Finally, in Sect. 6 we summarize our results and present our conclusions.

\section{Proposed system}

Considering the rapid proliferation of smart devices among travelers and passenger accommodation capacity in highspeed trains, an onboard distributed relay mechanism is proposed for HSR as shown in Fig. 2. The DMR nodes mounted on trains will act as relay nodes and equip the system to be resilient toward single-point failures. DMRs are further interfaced to the backhaul using WLAN Link (LTE-LAA) or LTE-Advanced.
Torch nodes (TNs) equipped in the front (TN-1) and rear (TN-2) of the train act as measurement nodes to provide prior information of donor eNBs (DeNBs) in a highly mobile environment. TNs report the measurement values to the onboard processing unit (OPU), which further uses this information for $\mathrm{HO}$ decision of respective DMRs. A global positioning system (GPS) integrated inertial measurement unit (IMU) is used to determine the speed of train. This unit is interfaced to OPU.

The OPU carries out two independent functionalities. The load balancer module of OPU effectively distributes the signaling load across multiple DMRs, whereas the measurement engine processes measurement details from TNs and DMRs based on speed of train and generate DMRspecific measurement report to be forwarded to respective DeNBs. OPU is connected with nodes using optical medium for faster data transfer and incorporates interface modules for TNs, DMRs, APs and IMU. Based on load sharing and network topology requirements, the OPU can be specific for each DMR node or a group of DMRs can share an OPU along with IMU. The block diagram of OPU is depicted in Fig. 3.

Based on the load being handled by respective DMRs, the OPU will dynamically link UEs to respective DMRs, thereby curbing the issue of overload. The onboard UEs are interfaced to DMRs via OPU through WLAN access points (APs) deployed across carriages.

When the train is at a higher speed, the measurement details of TNs will provide prior information of upcoming DeNBs to which HO needs to be initiated. Leveraging this information will provide sufficient lead time for DMRs in 


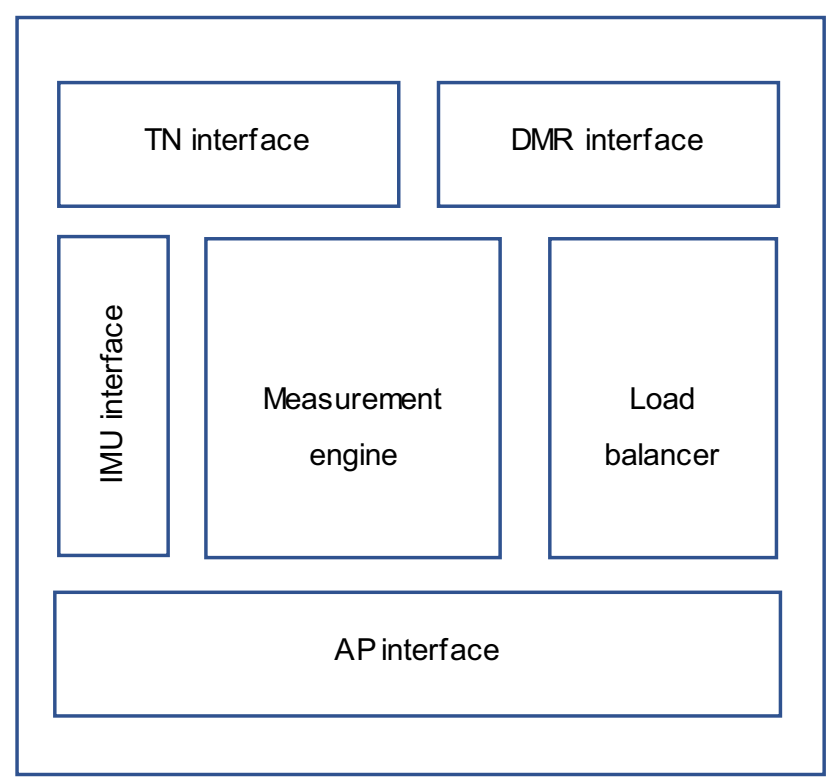

Fig. 3 Onboard processing unit

initiating and executing $\mathrm{HO}$ in a highly mobile HSR environment. At lower speeds or when the train is stationary, the signal strength information from respective DMR will be given preference while estimating measurement reports.

Based on velocity readings from IMU, the OPU provides a mobility-based adaptive measurement aggregation mechanism of values collected by TNs and DMRs to generate periodic- or event-based measurement report. This adaptive reporting strategy based on speed of train will facilitate DMRs to effectively speculate the upcoming DeNBs and initiate the HO early.

\section{Stages of proposed HO scheme}

The handover scheme proposed has the following three stages: (1) HO preparation, (2) HO execution and (3) HO completion.

\subsection{HO preparation stage}

LTE-Advanced adopts a UE assistance-based networkcontrolled hard HO procedure. In the context of HSR, the relay nodes deployed in train will take over the connectivity aspect from UEs, by acting as enablers between UE and DeNB as shown in Fig. 4. The DMRs will start its measurement procedure after receiving a measurement control command from the serving DeNB (S-DeNB). The DMRs will forward this measurement control command to OPU for configuring the TNs and IMU for initiating measurement in those nodes.

The measurement of DMRs, TNs and IMU will be forwarded as per pre-configured interval to the measurement engine module of OPU. This module implements an AMA mechanism based on the speed of train. Priority toward DMR measurement will be given at a time wherein the train is stationary or at lower speed. When the train attains moderate or higher speeds, AMA mechanism will give preference to readings from TNs, thereby facilitating an early reporting mechanism. This will provide

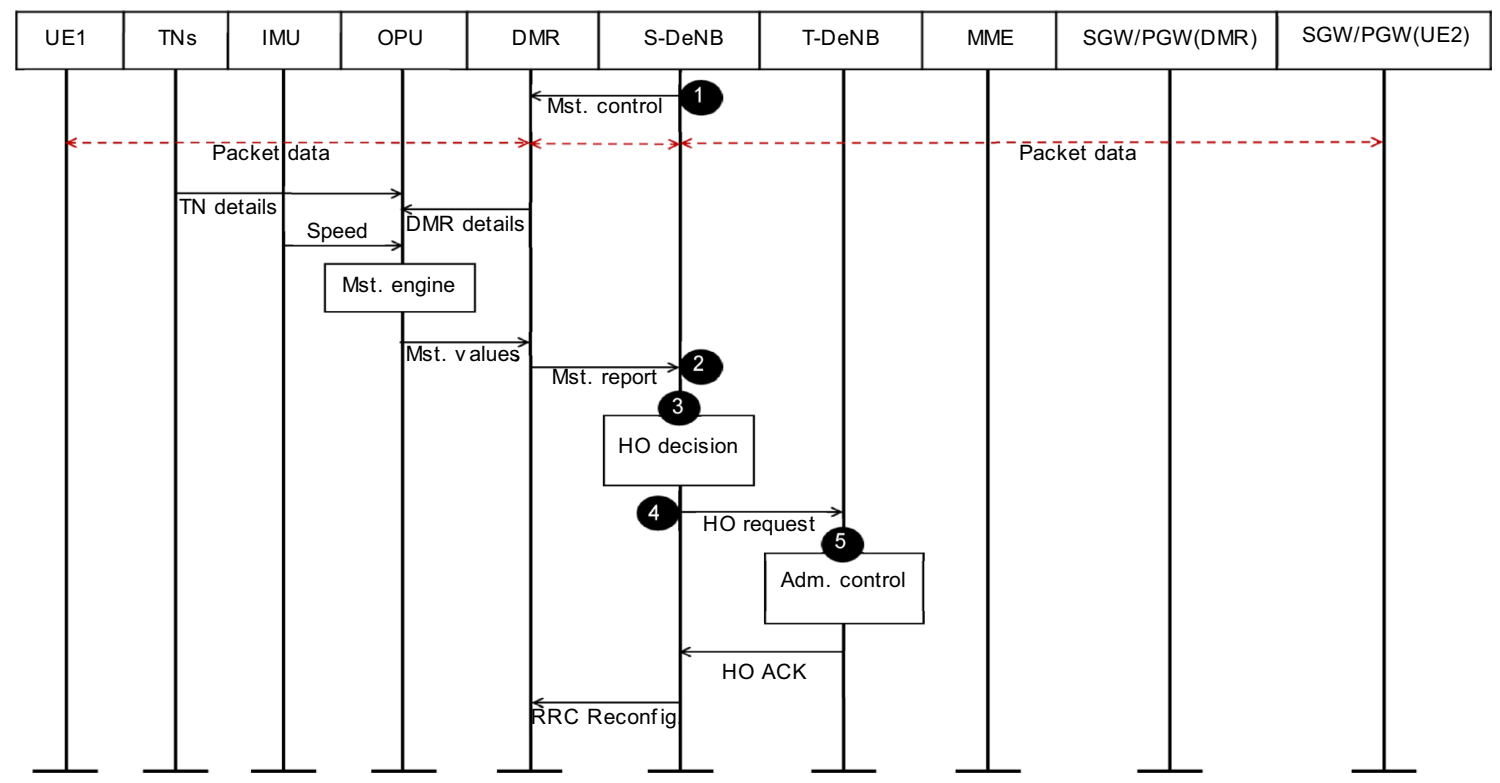

Fig. 4 Handover preparation stage 


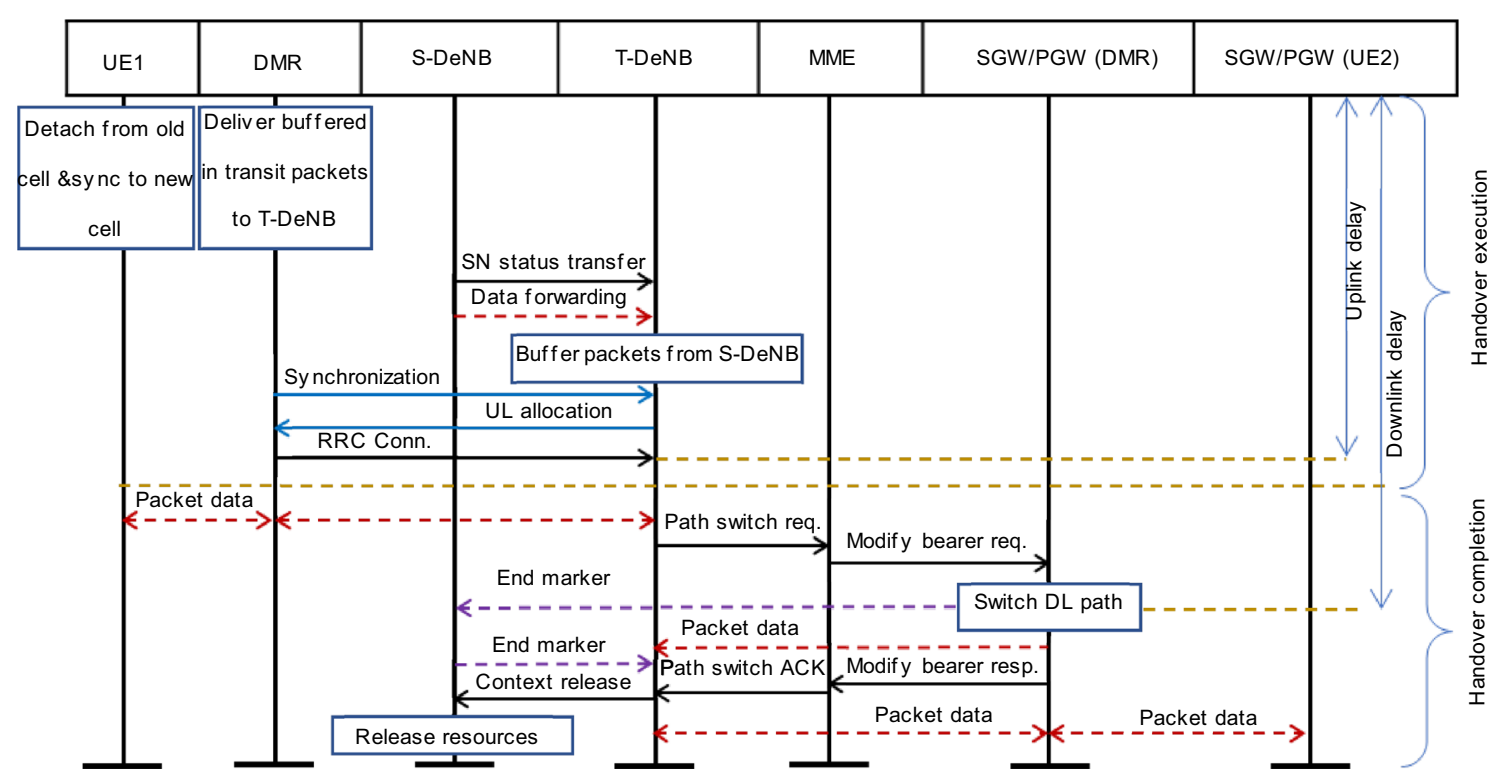

Fig. 5 Handover execution and completion stage

E-UTRAN and EPC with sufficient lead time in completing the HO procedures.

The OPU/DMR can be configured for periodic- or event-based reporting as per the measurement control command provided. Periodic configuration ensures that the OPU/DMR will report the measured signal strength values of target DeNB (T-DeNB) to S-DeNB in a fixed report interval (RI). S-DeNB performs $\mathrm{HO}$ when the signal quality of T-DeNB is above a threshold for continuous $k$ reports, where the value of $k$ is defined by cellular operator. During event-based reporting, the measurement control command will carry the appropriate event types and time-to-trigger (TTT) values.

Based on the LTE-Advanced radio resource control (RRC) specification, A3, A4 and A5 are the three event types defined to trigger the measurement reports.

\subsection{Handover execution stage}

As soon as the DMR receives the RRC reconfiguration message, the device detaches from S-DeNB. Further, it uses the configuration details to synchronize with the T-DeNB by sending contention-free-based random access preamble message. At the same time, the S-DeNB forwards the buffered data and status transfer command to T-DeNB. Once successfully synchronized, the DMR sends a tracking area update to MME along with RRC connection configuration complete message. This will establish the uplink communication between DMR and T-DeNB.

\subsection{Handover completion stage}

Based on the tracking update message from DMR, the MME updates the DMR location and replies with a tracking area update accept command to the DMR. The T-DeNB will also send a path switch request message to inform the MME that the S-DeNB of the DMR has changed and the routing path needs to be modified. Then, the MME issues a user plane update request command to S-GW to switch the routing path. A successful update will result in S-GW replying with a user plane update response command to the MME and MME further notifying T-DeNB using a path switch request acknowledgement (ACK). This would enable the T-DeNB and S-GW to forward data to DMR along the new path. Further, T-DeNB sends a context release message to request S-DeNB in releasing the context of DMR. This will complete the HO process. HO execution and completion stages are depicted in Fig. 5.

As observed, the proposed methodology will only modify the HO preparation stage of LTE-Advanced for reducing latency and improving the $\mathrm{HO}$ failure rates. Due to this strategy, the proposed mechanism can be easily adopted in the existing implementation with little or no modification in the mobile relay-based infrastructure setup. By adopting an AMA-based HO foreseeing approach, the system adapts itself to the dynamic nature of HSR environment. 


\section{AMA mechanism}

The adaptive measurement aggregation is a mechanism with which the DMR decides the measurement parameters to be reported to the S-DeNB. The decision in this regard will give due consideration to the mobility aspects of train and implements a predictive approach at higher speeds by correlating the measurement values of TNs along with DMR measurements.

\subsection{Measurement parameters}

According to $3 \mathrm{GPP}$, reference signal received power (RSRP) and reference signal received quality (RSRQ) are the major DMR-related values which need to be considered as measurement parameters in LTE-Advanced.

The RSRP measurement, $R_{\mathrm{P}}$, is the linear average of the power contributions by cell-specific resource elements that carry reference signals within the measurement frequency bandwidth as shown in Eq. (1). For absolute RSRP, the requirement would vary from \pm 6 to $\pm 11 \mathrm{~dB}$, whereas a relative measurement requirement varies from \pm 2 to $\pm 3 \mathrm{~dB}$ for intra-frequency and widens to $\pm 6 \mathrm{~dB}$ for interfrequency measurement. The RSRP measurements are further mapped to integer numbers, whose value ranges from 0 to 97 before they are included in the RRC messages.

$R_{\mathrm{P}}=P_{\mathrm{TX}}-T_{\mathrm{LS}}-f(\mu, \sigma)$,

where $P_{\mathrm{TX}}$ is the transmission power of DeNB, $T_{\mathrm{LS}}$ is the path loss of the signal between DeNB and DMR and $f(\mu, \sigma)$ is the shadow fading which usually follows Gaussian distribution with mean $\mu$ being zero and variance $\sigma^{2}$. For illustration, the path loss in $\mathrm{dB}$ [34] for a line of sight DeNB-DMR to model the propagation environment is as follows:

$T_{\mathrm{LS}}=22 \times \lg d+28.0+20 \times \lg F_{R}, \quad 10 \mathrm{~m}<d<d_{\mathrm{BP}}$,

where $F_{R}$ is the frequency $(\mathrm{Hz}) ; d_{\mathrm{BP}}$ is the breakpoint distance; the distance $d$ between DMR and DeNB is estimated as

$d=\sqrt{\left(y_{t}-y_{i}\right)^{2}+\left(x_{t}-x_{i}\right)^{2}}$,

where $\left(x_{t}, y_{t}\right)$ describes the position of DMR at time $t$ and $\left(x_{i}, y_{i}\right)$ defines the location of $\mathrm{DeNB}_{i}$.

RSRQ is a cell-specific signal quality metric, expressed in $\mathrm{dB}$, which is defined as $N_{\mathrm{RB}} R_{\mathrm{P}} / R_{\mathrm{S}}$, where $N_{\mathrm{RB}}$ is the number of resource blocks over which the measurement is conducted, and $R_{\mathrm{S}}$ is the $\mathrm{RSSI}_{\mathrm{E}-\mathrm{UTRA}}$ parameter which represents the entire received power from all interference and thermal noise source. In LTE-Advanced, the RSRQ measurement is also mapped from $\mathrm{dBm}$ to an integer number ranging from 0 to 34 .

Since RSRQ measurement combines signal strength and interference levels, this parameter is significant in assisting the mobility management in E-UTRAN. Mathematically, as the RSRQ measurement is proportional to the RSRP and signal strength, the current implementation considers RSRQ as the key measurement parameter along with mobility to implement the AMA mechanism. This optimizes and provides a fast, simplified and efficient aggregation and reporting mechanism.

In order to implement an effective $\mathrm{HO}$ mechanism for HSR, parameters with regard to mobility and service quality should also be considered while generating measurement reports. Such multi-criteria approach will provide an effective solution to enhance HO efficiency and increase resource utilization. In the current effort, the implementation consideration with regard to service quality is beyond the scope and will be addressed through a separate paper.

\subsection{AMA based on HSR mobility}

In a realistic LTE-Advanced scenario, the network will combine multiple radio access technologies (RATs), frequencies and cell sizes for efficient deployment [35] and the UEs carry out parameter measurement for multi-RAT intra-/interfrequency mobility. For elaboration simplicity, we consider interfrequency E-UTRAN HO process in the current context, whereas the proposed system with minimal enhancement can also be employed for inter-RAT HO mechanism.

Measurement reporting in LTE-Advanced can be configured as periodic, event triggered or event-triggered periodic. Triggering events as defined by 3GPP for monitoring the quality of serving cell and target cell are given in Table 1 and are calculated based on the entering conditions as elaborated in TS 36.331 .

The triggering events as defined for LTE-Advanced are more suitable for low mobility scenarios, whereas their applicability in a highly mobility environment like HSR requires considerable modification. Along with the measurement parameters as observed from target and serving cells, it is indispensable that the system should also consider the velocity of high-speed train (HST) to dynamically adapt the triggering events in a highly mobile environment. Considering various mobility scenarios in HSR, the variation in event triggering based on HST speed, $S$, is identified as follows:

(1) Movement of HST at lower speed ( $\left.S_{\text {Low }}\right)$

When stationary or at a lower speed than a threshold level (for example $200 \mathrm{~km} / \mathrm{h}$ ), the event triggering is considered 
Table 1 Event triggers for measurement report

\begin{tabular}{ll}
\hline Event & Description \\
\hline Inter-LTE mobility & S-DeNB better than absolute threshold \\
A1 & S-DeNB worse than absolute threshold \\
A2 & T-DeNB better than offset relative to S-DeNB \\
A3 & T-DeNB better than absolute threshold \\
A4 & S-DeNB worse than one absolute threshold (AT1) and T-DeNB better than another absolute threshold (AT2) \\
A5 & T-DeNB better than offset relative to secondary DeNB \\
A6 & T-DeNB better than absolute threshold \\
Inter-RAT mobility & S-DeNB worse than one absolute threshold $\mathrm{AT}_{R 1}$ and T-DeNB better than other absolute threshold AT \\
B1 & B2
\end{tabular}

based on the standard LTE-Advanced mechanism as detailed in Table 1.

(2) Movement of HST at higher speed $\left(S_{\mathrm{High}}\right)$

At an increased mobility scenario wherein the value of $S_{\text {High }}$ increases beyond a threshold level (for example, $200 \mathrm{~km} / \mathrm{h}$ ), it is essential for relay nodes to foresee the parameters of upcoming DeNB's and correlate the data with the measurements of DeNB's which are left behind to have an effective $\mathrm{HO}$ decision. Considering the assumption that the DeNBs deployed in an HSR is directional and continuous, the TNs deployed in the front and back of the train provide sufficient visibility to the futuristic and past parametric information.

In order to exemplify the above, we denote by $R_{\mathrm{Q} 1-\mathrm{FTN}}$, $R_{\mathrm{Q} 1-\mathrm{RTN}}$ and $R_{\mathrm{Q} 1-\mathrm{DMR}}$ the RSRQ measurements as observed by front TN, rear TN and DMR for S-DeNB, respectively, and by $R_{\mathrm{Q} 2-\mathrm{FTN}}, R_{\mathrm{Q} 2-\mathrm{RTN}}$ and $R_{\mathrm{Q} 2-\mathrm{DMR}}$ the counterparts as reported for T-DeNB. A parameter pattern, $R_{\mathrm{Q} 1-\mathrm{RTN}} \geq$ $R_{\mathrm{Q} 1 \text {-DMR }} \geq R_{\mathrm{Q} 1 \text {-FTN }}$, being reported for $\mathrm{S}$-DeNB node observed for TTT duration, can be inferred to be a condition wherein the train is leaving the vicinity of a cell. Meanwhile, $R_{\mathrm{Q} 2 \text {-FTN }} \geq R_{\mathrm{Q} 2 \text {-DMR }}$ for the $\mathrm{T}$-DeNB node indicates that the train is entering the cell.

Considering this aspect, the $\mathrm{HO}$ event generated at $\mathrm{HO}$ initiate time for DMR-TN, $T_{\mathrm{HS} \text {-initiate, }}$ as shown in Eq. (4) provides a realistic and accurate $\mathrm{HO}$ decision-making scenario, in comparison with $\mathrm{HO}$ initiate time, $T_{\mathrm{HO}}$-initiate, as recommended by 3 GPP standard where event triggering occurs whenever the T-DeNB is better than the S-DeNB.

$$
\left(R_{\mathrm{Q} 1-\mathrm{RTN}}>R_{\mathrm{Q} 1-\mathrm{DMR}} \geq R_{\mathrm{Q} 1-\mathrm{FTN}}\right) \mathrm{AND}\left(R_{\mathrm{Q} 2-\mathrm{FTN}}>R_{\mathrm{Q} 2-\mathrm{DMR}}\right) .
$$

In such a scenario, it is ideal to have the $\mathrm{HO}$ initiated at

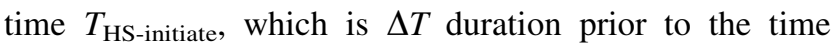
$T_{\text {HO-initiate }}$ as defined by the 3 GPP specification. This would provide sufficient lead time in initiating the $\mathrm{HO}$ procedure in a high mobility HSR scenario. Moreover, the proposed method envisages significant reduction in the time for DMR to reenter ( $\left.T_{\text {Reentry }}\right)$ and the time to re-establish the corresponding DMR connection during call drop ( $\left.T_{\text {Reconnect }}\right)$

$T_{\mathrm{HS}-\text { initiate }}=T_{\mathrm{HO} \text {-initiate }}-\Delta T$.

In contrast to the conventional approach of taking measurement parameters from a single MRN, it is essential to adapt to the varied mobility conditions and estimate the parameter values dynamically. Though various approaches can be applied to generate events as mentioned in Eq. (4), a weighted average mechanism to aggregate the S-DeNB and T-DeNB measurements across front TN (TN-1), rear TN (TN-2) and DMR is adopted here as shown in Eq. (6). The weights are determined on the basis of mobility conditions of HST.

$R_{\mathrm{Q}-\mathrm{MN}}=W_{1} \cdot R_{\mathrm{Q}-\mathrm{DMR}}+W_{2} \cdot R_{\mathrm{Q}-\mathrm{FTN}}+W_{3} \cdot R_{\mathrm{Q}-\mathrm{RTN}}$,

where $R_{\mathrm{Q}-\mathrm{MN}}$ is the aggregate RSRQ measurement to be reported for event triggering, and $W_{i}(i=1,2,3)$ is the significance parameter.

\section{Simulation and performance analysis}

\subsection{Implementation of simulation model}

The proposed model was designed and implemented on an object-oriented discrete event simulator [36] extended with an LTE-Advanced package [37]. A representative illustration of the simulation environment on Omnet++ involving a train traveling in an HSR environment between two stations is presented in Fig. 6a. The simulated infrastructure consists of DeNBs, Routers, P-GW and Server. The DeNBs uses omnidirectional anisotropic antennas and are interconnected through $\mathrm{X} 2$ interface. 

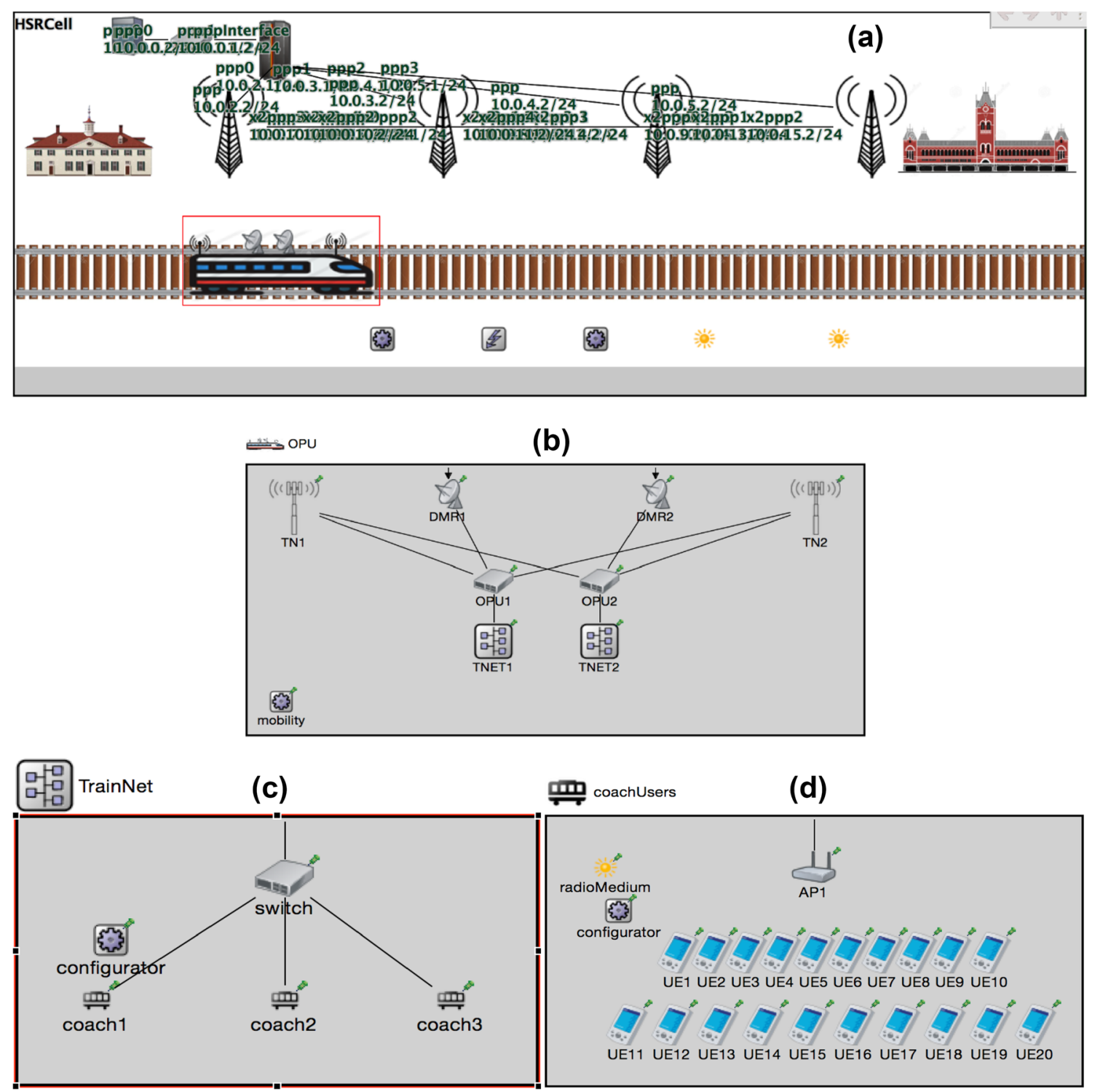

Fig. 6 Omnet ++ simulation screenshot. a HSR simulation between two stations. b Simulated onboard train network. c LAN network across coaches. d Wireless LAN connectivity at coaches

As shown in Fig. 6b, the implementation of onboard train network involves front torch nodes (TN-1), rear torch nodes (TN-2), two distributed mobile relay nodes (DMR-1 and DMR-2), onboard processing units (OPU1 and OPU2) and train networks (TNET1 and TNET2). Figure 6c shows the implementation of TNET which establishes LAN connectivity across multiple coaches. TNETs are further interfaced to the UEs through wireless APs as shown in Fig. 6d. IMU unit was implemented as a module in OPU, and implementation of load balancer was simplified to handle individual DMR. Table 2 summarizes the simulation parameters. The simulation uses ITU Urban Macro [38] for path loss and Jakes fading model [39].

\subsection{Simulation results}

We validated the model and carried out simulations to assess the performance of the proposed system in improving the HO latency and HO failure probability. Performance of the proposed model is compared with that of the single link mobile wireless relay node (MRN). The MRN uses only single wireless link to connect to DeNB, in comparison with the proposed DMR-TN system, which involves TNs for efficient estimation of measurements in a mobile environment, multiple antenna scheme to enhance the load distribution, enhanced monitoring using AMA and efficient message handling capabilities. 
Table 2 Simulation parameters

\begin{tabular}{ll}
\hline Parameters & Values \\
\hline DeNB & Single cell base station \\
User equipment (UE) & $120 \mathrm{Nos}$. \\
Macrocell radius & $1500 \mathrm{~m}$ \\
Macrooverlapped area & $400 \mathrm{~m}$ \\
Railway distance & $7000 \mathrm{~m}$ \\
Speed of train & $200-400 \mathrm{~km} / \mathrm{h}$ \\
Length of train & $200 \mathrm{~m}$ \\
Application & VOIP \\
Carrier frequency & $2 \mathrm{GHz}$ \\
Bandwidth & $5 \mathrm{MHz}(25 \mathrm{RBs})$ \\
Path loss model & ITU Urban Macro \\
DeNBTx power & $46 \mathrm{~dB}$ \\
Mobility model & Linear mobility \\
Simulation time & $75 \mathrm{~s}$ \\
\hline
\end{tabular}

\section{(1) HO latency}

The HO latency is computed from the time when the SDeNB begins its HO to the time when the connection is established for data transmission to T-DeNB. The HO latency $T_{\mathrm{HO}-\text { latency }}$ is estimated as follows:

$$
\begin{aligned}
T_{\mathrm{HO}-\text { latency }}= & P_{\mathrm{SHO}} \cdot T_{\mathrm{HO}} \\
& +\left(1-P_{\mathrm{SHO}} \cdot\left(T_{\text {Reentry }}+T_{\text {Reconnect }}\right),\right.
\end{aligned}
$$

where the $T_{\mathrm{HO}}$ is the time taken to handover; $P_{\mathrm{SHO}}$ is the probability estimate value for calculating the average $\mathrm{HO}$ latency, and is considered based on [26, 40]. The probability estimate of successful handover for MRN scheme, $P_{\text {SHO-MRN }}$, is derived as 0.97 , and the probability estimate of successful handover for DMR-TN, $P_{\text {SHO-DMR-TN, }}$ is 0.999. The $\mathrm{HO}$ in the proposed approach significantly reduces the $T_{\text {Reentry }}$ and $T_{\text {Reconnect }}$ and increases the probability of success at each stage. The onboard measurement analyzer and multi-antenna with load balancer have reduced the $\mathrm{HO}$ latency in comparison with the existing systems.

Figure 7 illustrates the HO latency of the simulation run wherein the proposed DMR-TN scheme outperforms the single-antenna-based MRN scheme. Although users with high mobility are expected to experience an increase in $\mathrm{HO}$ latency, the HO latency in the proposed solution is comparatively smaller and increases gradually as the speed increases.

\section{(2) HO failure probability}

Once $\mathrm{HO}$ is triggered, any subsequent degradation of T-DeNB signal strength below a threshold level will result in connection losses and an $\mathrm{HO}$ failure will occur if this interruption exceeds the predefined delay limit. In the proposed scheme, the measurement report from TNs provides a further assurance in estimating the T-DeNB, resulting in significant reduction in $\mathrm{HO}$ failures. Figure 8 shows that the HO failure probability of the simulated model is on an average of 0.01 , whereas the value is near to 0.05 for MRN scheme.

\section{(3) Communication interruption probability}

The probability of communication interruption (CI) during an $\mathrm{HO}$ procedure provides a detailed overview of the efficacy of a model. For a system to provide improved QoS and seamless user experience, the communication interruption probability needs to be smaller. In the simulation model, we plotted the CI probability as a function of $\mathrm{HO}$ locations within a range of $1100-1500-\mathrm{m}$ region in the overlap area. In comparison with the single-antenna MRN model, the interruption probability for the proposed DMRTN scheme is less than 0.1 for the majority of overlap area, as shown in the simulation results given in Fig. 9. The involvement of torch nodes and adaptive measurement aggregation mechanism module has significantly

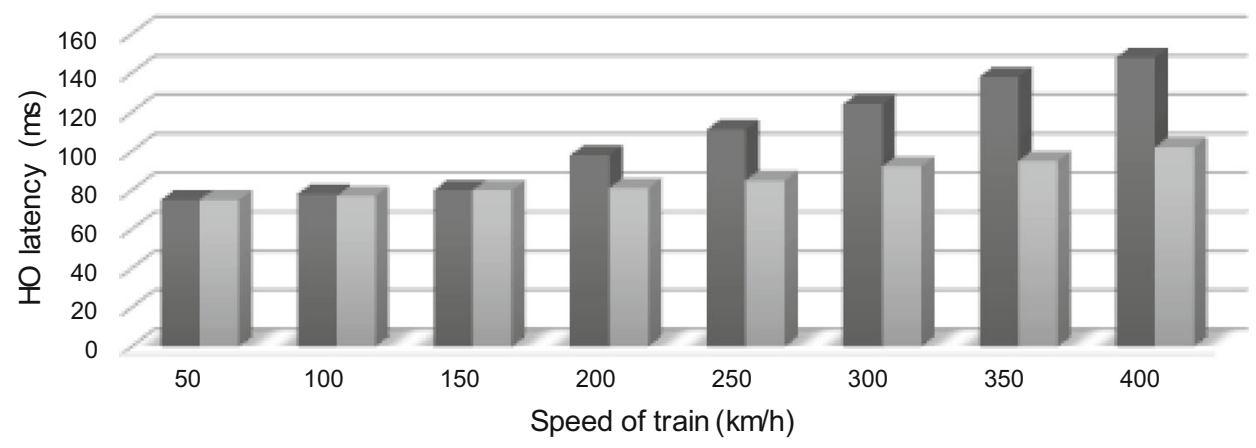

$\because \mathrm{MRN} \backsim \mathrm{DMR}-\mathrm{TN}$

Fig. 7 HO latency 


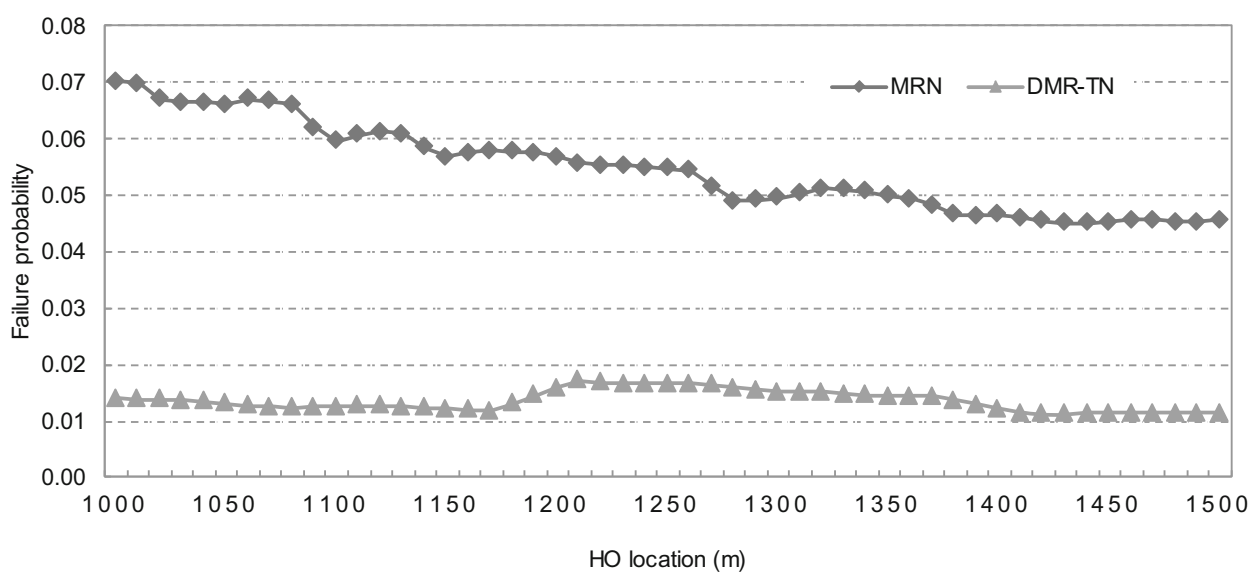

Fig. 8 HO failure probability

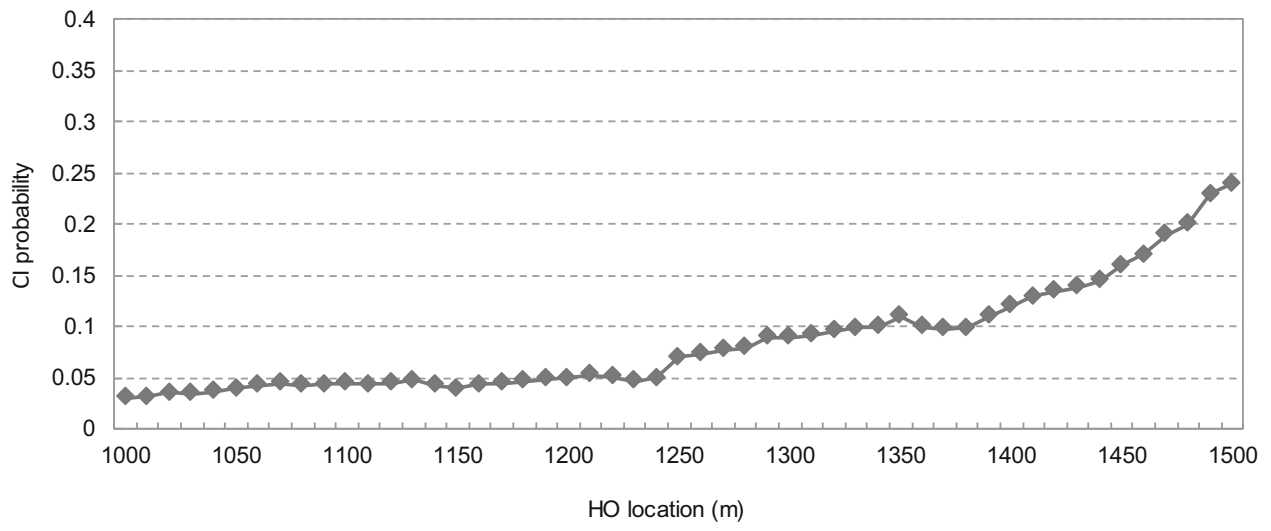

Fig. 9 Communication interruption probability for the proposed DMR-TN scheme

contributed in decreasing the failure probability as the distance increases.

\section{Conclusion and future work}

This paper presents the details of a novel scheme to improve QoS in a high-speed railway communication system. The proposed scheme involves the following key features: (1) torch nodes to effectively forecast target DeNBs, (2) adaptive measurement aggregation based on mobility and (3) distributed mobile relays with load balancer for efficient resource utilization. The proposed scheme was simulated using Omnet ++ tool, and the performance is analyzed in terms of handover latency, handover failure probability and communication interruption probability. The preliminary analysis shows that the DMRTN scheme outperforms the single-antenna scheme and provides better performance with reduced handover failure and communication interruption probability. The proposed scheme can be easily implemented in high-speed railways with reduced implementation overhead. In the future work, we plan to carryout further optimization by considering service-based parameters (e.g., voice, video, and data) to enhance handover efficiency and resource optimization.

Acknowledgements The authors would like to thank Centre for Development of Advanced Computing (C-DAC), Hyderabad, and Jawaharlal Nehru Technological University, Hyderabad, in facilitating this research work.

Open Access This article is distributed under the terms of the Creative Commons Attribution 4.0 International License (http:// creativecommons.org/licenses/by/4.0/), which permits unrestricted use, distribution, and reproduction in any medium, provided you give appropriate credit to the original author(s) and the source, provide a link to the Creative Commons license, and indicate if changes were made. 


\section{References}

1. Saleh AAM, Simmons JM (2011) Technology and architecture to enable the explosive growth of the internet. IEEE Commun Mag 49(1):126-132. https://doi.org/10.1109/MCOM.2011.5681026

2. Corcoran P (2017) Mobile-edge computing and internet of things for consumers: part II: energy efficiency, connectivity, and economic development. IEEE Consum Electron Mag 6(1):51-52. https://doi.org/10.1109/MCE.2016.2614418

3. GSMA Intelligence (2016) Global mobile trends. Study Report

4. Garcia Alvarez A (2014) The advantages and advances of electric railways [viewpoint]. IEEE Electr Mag 2(3):60-64. https://doi. org/10.1109/MELE.2014.2339411

5. Xiaowen Fu, Zhang Anming, Lei Zheng (2012) Will China's airline industry survive the entry of high-speed rail? Res Trans Econ 35(1):13-25. https://doi.org/10.1016/j.retrec.2011.11.006

6. Akerman Jonas (2011) The role of high-speed rail in mitigating climate change-The Swedish case Europabanan from a life cycle perspective. Transp Res Part D Trans Environ 16(3):208-217. https://doi.org/10.1016/j.trd.2010.12.004

7. Cascetta Ennio, Papola Andrea, Pagliara Francesca, Marzano Vittorio (2011) Analysis of mobility impacts of the high speed Rome-Naples rail link using within day dynamic mode service choice models. J Transp Geogr 19(4):635-643. https://doi.org/10. 1016/j.jtrangeo.2010.07.001

8. Chen Chia-Lin, Hall Peter (2011) The impacts of high-speed trains on British economic geography: a study of the UK's InterCity 125/225 and its effects. J Transp Geogr 19(4):689-704. https://doi.org/10.1016/j.jtrangeo.2010.08.010

9. Hang Z (2011) The impact of the high-speed train in China. Int Conf Manag Serv Sci Wuhan. https://doi.org/10.1109/ICMSS. 2011.5998237

10. 3rd Generation Partnership Project (2009) 3GPP-TR-25.913 Requirements for evolved UTRA (E-UTRA) and evolved UTRAN (E-UTRAN). Technical Report

11. 3rd Generation Partnership Project (2015) 3GPP-TR-36.913 Requirements for further advancements for Evolved Universal Terrestrial Radio Access (E-UTRA) (LTE-Advanced). Technical Report

12. Akyildiz Ian F, Gutierrez-Estevez David M, Reyes Elias Chavarria (2010) The evolution to 4G cellular systems: 1TEAdvanced. Phys Commun 3(4):217-244. https://doi.org/10.1016/ j.phycom.2010.08.001

13. Agilent Tech (2011) Introducing LTE-advanced. Application note

14. Ali-Yahiya Tara (2011) Understanding LTE and its Performance. Springer-Verlag, New York. https://doi.org/10.1007/978-1-44196457-1. ISBN 978-1-4419-6457-1

15. 3rd Generation Partnership Project (2016) 3GPP-TR-36.300 Evolved Universal Terrestrial Radio Access (E-UTRA) and Evolved Universal Terrestrial Radio Access Network (EUTRAN)-Overall description. Technical specification

16. Youping Zhao and Jinxing Li (2011) Analysis of the impact of Doppler spread on OFDM-based next-generation high-speed rail broadband mobile communications. IET Int Conf Commun Technol Appl Beijing. https://doi.org/10.1049/cp.2011.0642

17. Ghazzai H, Bouchoucha T, Alsharoa A, Yaacoub E, Alouini MS, Al-Naffouri TY (2017) Transmit power minimization and base station planning for high-speed trains with multiple moving relays in OFDMA systems. IEEE Trans Veh Technol 66(1):175-187. https://doi.org/10.1109/TVT.2016.2542344

18. Lin X, Xu C, Zhang Siyu, Zhao Ruojun and Xiao Xiang (2014) Comparative analysis on fading characteristics of the LTE-R channel in viaduct and hilly terrain scenarios. In: International symposium on wireless personal multimedia communications (WPMC), Sydney https://doi.org/10.1109/WPMC.2014.7014804

19. He R, Zhong Z, Ai B, Ding J, Jiang W, Zhang H, and Li X (2014) A standardized path loss model for the GSM-Railway based highspeed railway communication systems. In: IEEE 79th vehicular technology conference (VTC Spring), Seoul https://doi.org/10. 1109/VTCSpring.2014.7022797

20. Sun W, Wang H, Bu B (2011) Feasibility research on trainground information transmission based on leaky coaxial cable in CBTC. In: Proceedings of 2011 IEEE international conference on service operations, logistics and informatics Beijing. https://doi. org/10.1109/SOLI.2011.5986626

21. Lannoo B, Colle D, Pickavet M, Demeester P (2007) Radio-overFiber based solution to provide broadband internet access to train passengers [topics in optical communications]. IEEE Commun Mag 45(2):56-62. https://doi.org/10.1109/MCOM.2007.313395

22. Kawanishi T, Kanno A, Dat PT and Yamamoto N (2016) Seamless access networks using radio-over-fiber technology for high-speed trains. In: IEEE Sixth international conference on communications and electronics (ICCE), Ha Long. https://doi. org/10.1109/CCE.2016.7562666

23. Haddad A and Gagnaire M (2014) Radio-over-Fiber (RoF) for mobile backhauling: a technical and economic comparison between analog and digitized RoF. In: International conference on optical network design and modeling, Stockholm

24. Hou HA and Wang LC (2015) A systematic approach to improve fiber-wireless access network in high speed railway tunnels. In: IEEE international conference on communication workshop (ICCW), London. https://doi.org/10.1109/ICCW.2015.7247196

25. Han M, Lee N, Han K, Lee D (2008) Fast IP handover between satellite networks and wireless LAN networks for high-speed trains. IP Netw Over Next-Gener Satell Syst Springer, New York. https://doi.org/10.1007/978-0-387-75428-4_4

26. Tian L, Li J, Huang Y, Shi J, Zhou J (2012) Seamless dual-link handover scheme in broadband wireless communication systems for high-speed rail. IEEE J Sel Areas Commun 30(4):708-718. https://doi.org/10.1109/JSAC.2012.120505

27. 3rd Generation Partnership Project (2014) 3GPP-TR-36.836 Evolved Universal Terrestrial Radio Access (E-UTRA); Study on Mobile Relay. Technical Report

28. Chen L, Huang Y, Xie F, Gao Y, Chu L, He H, Li Y, Liang F, Yuan Y (2013) Mobile relay in LTE-advanced systems. IEEE Commun Mag 51(11):144-151. https://doi.org/10.1109/MCOM. 2013.6658666

29. Li W, Zhang C, Duan X, Jia S, Liu Y and Zhang L (2012) Performance evaluation and analysis on group mobility of mobile relay for LTE advanced system. In: IEEE vehicular technology conference (VTC Fall), Quebec City. https://doi.org/10.1109/ VTCFall.2012.6399277

30. Alsharoa A, Ghazzai H, Yaacoub E and Alouini MS (2014) Energy-efficient two-hop LTE resource allocation in high speed trains with moving relays. In: International symposium on modeling and optimization in mobile, Ad Hoc, and Wireless Networks (WiOpt), Hammamet. https://doi.org/10.1109/WIOPT. 2014.6850342

31. Liu Z, Fan P (2014) An effective handover scheme based on antenna selection in ground-train distributed antenna systems. IEEE Trans Veh Technol 63(7):3342-3350. https://doi.org/10. 1109/TVT.2014.2300154

32. Deng T, Zhang Z, Wang X and Fan P (2016) A network assisted fast handover scheme for high speed rail wireless networks. In: IEEE 83rd vehicular technology conference (VTC Spring), Nanjing. https://doi.org/10.1109/VTCSpring.2016.7504083

33. Pan MS, Lin TM, Chen WT (2015) An enhanced handover scheme for mobile relays in lte-a high-speed rail networks. IEEE 
Trans Veh Technol 64(2):743-756. https://doi.org/10.1109/TVT. 2014.2322374

34. Wang H, Rosa C, Pedersen KI (2016) Dual connectivity for LTEadvanced heterogeneous networks. K.I. Wireless Networks 22(4):1315-1328. https://doi.org/10.1007/s11276-015-1037-6

35. Munoz P, Barco R, Laselva D, Mogensen P (2013) Mobilitybased strategies for traffic steering in heterogeneous networks. IEEE Commun Mag 51(5):54-62. https://doi.org/10.1109/ MCOM.2013.6515047

36. AndrásVarga and Rudolf Hornig. (2008) An overview of the $\mathrm{OMNeT}++$ simulation environment. In: Proceedings of the 1 st international conference on Simulation tools and techniques for communications, networks and systems \& workshops (Simutools '08) Belgium

37. Virdis A, Stea G and Nardini G (2014) SimuLTE-A modular system-level simulator for LTE/LTE-A networks based on
OMNeT++. 4th International Conference on Simulation And Modeling Methodologies, Technologies And Applications (SIMULTECH), Vienna, Austria. https://doi.org/10.5220/ 0005040000590070

38. 3rd Generation Partnership Project (2010) TR-36.814 Further advancements forE-UTRA physical layer aspects. Technical Report

39. Jakes William C (1994) Microwave Mobile Communications. An IEEE Press Classic Reissue, Wiley. ISBN 978-0-7803-1069-8

40. Lee CW, Chuang MC, Chen MC, Sun YS (2014) Seamless Handover for high-speed trains using femtocell-based multiple egress network interfaces. IEEE Trans Wireless Commun 13(12):6619-6628. https://doi.org/10.1109/TWC.2014.2364179 